\title{
Hyers-Ulam stability of functional inequalities: a fixed point approach
}

\author{
Afshan Batool ${ }^{1}$, Sundas Nawaz ${ }^{2}$, Ozgur Ege ${ }^{3^{*}}$ (I) and Manuel de la Sen ${ }^{4}$
}

"Correspondence:

ozgur.ege@ege.edu.tr

${ }^{3}$ Department of Mathematics, Faculty of Science, Ege University, Bornova, 35100, Izmir, Turkey Full list of author information is available at the end of the article

\begin{abstract}
Using the fixed point method, we prove the Hyers-Ulam stability of a cubic and quartic functional equation and of an additive and quartic functional equation in matrix Banach algebras.
\end{abstract}

MSC: Primary 47H10; secondary 39B52; 39B72

Keywords: Functional inequality; Additive mapping; Fixed point; Matrix Banach algebra; Hyers-Ulam stability

\section{Introduction and preliminaries}

Ulam [30] raised a question concerning the stability of group homomorphisms. The functional equation

$$
f(a+b)=f(a)+f(b)
$$

is familiar as a Cauchy equation, in particular, every solution of a Cauchy equation is called an additive mapping. Hyers [15] gave the first answer to the question of Ulam for Banach spaces as follows.

Theorem 1.1 Let $X$ and $Y$ be Banach spaces. Assume that $f: X \rightarrow Y$ satisfies

$$
\|f(x+y)-f(x)-f(y)\| \leq \varepsilon
$$

for all $x, y \in X$ and some $\varepsilon \geq 0$. Then there exists a unique additive mapping $T: X \rightarrow Y$ such that $\|f(x)-T(x)\| \leq \varepsilon$ for all $x \in X$.

Hyers' theorem was generalized by Aoki [1] for additive mappings and by Rassias [28] for linear mappings by considering an unbounded Cauchy difference. A generalization of Rassias' theorem was given by Gavruta [13] by replacing the unbounded Cauchy difference with a general control function. In 1982, Rassias [24] after the innovative approach of the Rassias' theorem [28] replaced $\|x\|^{p}+\|y\|^{p}$ by $\|x\|^{p} \cdot\|y\|^{q}$ for $p, q \in \mathbb{R}$ with $p+q \neq 1$. A generalization of Hyers-Ulam stability problem for the quadratic functional equation

(c) The Author(s) 2020. This article is licensed under a Creative Commons Attribution 4.0 International License, which permits use, sharing, adaptation, distribution and reproduction in any medium or format, as long as you give appropriate credit to the original author(s) and the source, provide a link to the Creative Commons licence, and indicate if changes were made. The images or other third party material in this article are included in the article's Creative Commons licence, unless indicated otherwise in a credit line to the material. If material is not included in the article's Creative Commons licence and your intended use is not permitted by statutory regulation or exceeds the permitted use, you will need to obtain permission directly from the copyright holder. To view a copy of this licence, visit http://creativecommons.org/licenses/by/4.0/ 
was given by Skof [29] for mappings $f: X \rightarrow Y$, where $X$ is a normed space and $Y$ is a Banach space. Cholewa [10] noticed that the theorem of Skof is still true if the relevant domain $X$ is replaced by an abelian group. Czerwik [11] proved the Hyers-Ulam stability of the quadratic functional equation. The stability problem of functional equations has been discussed by many mathematicians using different spaces and mappings. Park and Najati [22] proved the Hyers-Ulam stability of functional equations in real Banach spaces. The stability problems of several functional equations have been extensively investigated by a number of authors and there are many interesting results concerning this problem (see $[2,3,5,16,21,24]$ ).

In $[26,27]$, Rassias first introduced and investigated the cubic functional equation

$$
f(x+2 y)+3 f(x)=3 f(x+y)+f(x-y)+6 f(y) .
$$

In [18], Jun and Kim considered the following cubic functional equation:

$$
f(2 x+y)+f(2 x-y)=2 f(x+y)+2 f(x-y)+12 f(x) .
$$

It is easy to show that the function $f(x)=x^{3}$ satisfies the functional equation (1.1) and every solution of the cubic functional equation is said to be a cubic mapping. Rassias [25] first introduced and investigated the quartic functional equation.

$$
f(2 x+y)+f(2 x-y)=4 f(x+y)+4 f(x-y)+24 f(x)-6 f(y)
$$

and Lee et al. [20] investigated the quartic functional equation (1.2). It is easy to show that the function $f(x)=x^{4}$ satisfies the functional equation (1.2) and every solution of the quartic functional equation is said to be a quartic mapping.

We recall a fundamental result in fixed point theory. For some recent papers on fixed point theory, see $[4,6,14,19]$.

Theorem $1.2([7,12])$ Let $(U, d)$ be a complete generalized metric space and $J: U \rightarrow U$ be a strictly contractive mapping with Lipschitz constant $L<1$. Then, for each given element $x \in U$, either

$$
d\left(J^{n} x, J^{n+1} x\right)=\infty
$$

for all nonnegative integers $n$ or there is a positive integer $n_{0}$ such that

(1) $d\left(J^{n} x, J^{n+1} x\right)<\infty$ for all $n \geq n_{0}$;

(2) the sequence $\left\{J^{n} x\right\}$ converges to a fixed point $y^{*}$ of $J$;

(3) $y^{*}$ is the unique fixed point of $J$ in the set $Y=\left\{y \in U \mid d\left(J^{n_{0}} x, y\right)<\infty\right\}$;

(4) $d\left(y, y^{*}\right) \leq \frac{1}{1-L} d(y, J y)$ for all $y \in Y$.

We will use the following notations:

- $M_{n}(U)$ is the set of all $n \times n$-matrices in $U$;

- $e_{j} \in M_{1, n}(\mathbb{C})$ means that $j$ th component is 1 and the other components are zero;

- $E_{i j} \in M_{n}(\mathbb{C})$ is that the $(i, j)$-component is 1 and the other components are zero;

- $E_{i j} \otimes x \in M_{n}(\mathbb{C})$ means that the $(i, j)$-component is $x$ and the other components are zero; 
- for $x \in M_{n}(U), y \in M_{n}(U)$,

$$
x \oplus y=\left(\begin{array}{ll}
x & 0 \\
0 & y
\end{array}\right) .
$$

Note that $\left(U,\|\cdot\|_{n}\right)$ is a matrix normed space if and only if $\left(M_{n}(U),\|\cdot\|_{n}\right)$ is a normed space for each positive integer $n$ and

$$
\|A x B\|_{k} \leq\|A\|\|B\|\|x\|_{n}
$$

holds for $A \in M_{n}(\mathbb{C}), x=\left[x_{i j}\right] \in M_{n}(\mathbb{C})$ and $\left.B \in M_{n, k}(\mathbb{C})\right)$ and that $\left(U,\|\cdot\|_{n}\right)$ is a matrix Banach space if and only if $U$ is a Banach space and $\left(U,\|\cdot\|_{n}\right)$ is a matrix normed space. A matrix Banach space $\left(U,\|\cdot\|_{n}\right)$ is called a matrix Banach algebra if $U$ is an algebra. A matrix normed space $\left(U,\|\cdot\|_{n}\right)$ is called an $L^{\infty}$-matrix normed space if

$$
\|x \oplus y\|_{n+k}=\max \left\{\|x\|_{n},\|y\|_{k}\right\}
$$

holds for all $x \in M_{n}(U), y \in M_{k}(U)$.

Let $E, F$ be vector spaces. For a given mapping $h: E \rightarrow F$ and a given positive integer $n$, define $h_{n}: M_{n}(E) \rightarrow M_{n}(F)$ by

$$
h_{n}\left(\left[x_{i j}\right]\right)=\left[h\left(x_{i j}\right)\right]
$$

for all $\left[x_{i j}\right] \in M_{n}(E)$.

Lemma 1.3 Let $\left(U,\|\cdot\|_{n}\right)$ be a matrix normed space.

- $\left\|E_{k l} \otimes x\right\|_{n}=\|x\|$ for $x \in U$.

- $\left\|x_{k l}\right\| \leq\left\|\left[x_{i j}\right]\right\|_{n} \leq \sum_{i, j=1}^{n}\left\|x_{i j}\right\|$ for $\left[x_{i j}\right] \in M_{n}(U)$.

- $\lim _{n \rightarrow \infty} x_{n}=x$ if and only if $\lim _{n \rightarrow \infty} x_{n i j}=x_{i j}$ for $x_{n}=\left[x_{i j}\right], x=\left[x_{i j}\right] \in M_{k}(U)$.

This paper is organized as follows: In Sects. 2 and 3, using the fixed point method, we prove the Hyers-Ulam stability of the cubic and quartic functional equation

$$
\begin{aligned}
f(2 x+y)+f(2 x-y)= & 3 f(x+y)+f(-x-y)+3 f(x-y)+f(y-x) \\
& +18 f(x)+6 f(-x)-3 f(y)-3 f(-y)
\end{aligned}
$$

in matrix Banach algebras. In Sects. 4 and 5, using the fixed point method, we prove the Hyers-Ulam stability of the additive and quartic functional equation

$$
\begin{aligned}
f(2 x+y)+f(2 x-y)= & 2 f(x+y)+2 f(-x-y)+2 f(x-y)+2 f(y-x) \\
& +14 f(x)+10 f(-x)-3 f(y)-3 f(-y)
\end{aligned}
$$

in matrix Banach algebras.

In 1996, Rassias and Isac [17] were the first to provide applications of stability theory of functional equations for the proof of new fixed point theorems with applications. By using 
the fixed point method, the stability problems of several functional equations have been extensively investigated by a number of authors (see $[9,23])$.

Throughout this paper, we assume that $X$ is a matrix normed space and that $Y$ is a matrix Banach algebra.

\section{Fixed points and Hyers-Ulam stability of a cubic and quartic functional equation: an even case}

One can easily show that an even mapping $f: X \rightarrow Y$ satisfies (1.3) if and only if the even mapping $f: X \rightarrow Y$ is a quartic mapping, i.e.,

$$
f(2 x+y)+f(2 x-y)=4 f(x+y)+4 f(x-y)+24 f(x)-6 f(y)
$$

and that an odd mapping $f: X \rightarrow Y$ satisfies (1.3) if and only if the odd mapping $f: X \rightarrow Y$ is a cubic mapping, i.e.,

$$
f(2 x+y)+f(2 x-y)=2 f(x+y)+2 f(x-y)+12 f(x) .
$$

It is easy to show that the function $f(x)=a x^{3}+b x^{4}$ satisfies the functional equation (1.3).

For a given mapping $f: X \rightarrow Y$, we define

$$
\begin{aligned}
D f(x, y):= & f(2 x+y)+f(2 x-y)-3 f(x+y)-f(-x-y) \\
& -3 f(x-y)-f(y-x)-18 f(x)-6 f(-x)+3 f(y)+3 f(-y)
\end{aligned}
$$

for all $x, y \in \mathrm{X}$.

Using the fixed point method, we prove the Hyers-Ulam stability of the functional equation $D f(x, y)=0$ in matrix Banach algebras: an even case.

Theorem 2.1 Let $f: X \rightarrow Y$ be a mapping with $f(0)=0$ for which there exists a function $\varphi: X^{2} \rightarrow[0, \infty)$ such that there exists an $L<1$ satisfying

$$
\begin{aligned}
& \sum_{i, j=1}^{n} \varphi\left(x_{i j}, y_{i j}\right) \leq \sum_{i, j=1}^{n} \frac{1}{16} L \varphi\left(2 x_{i j}, 2 y_{i j}\right), \\
& \left\|D f_{n}\left(\left[x_{i j}\right],\left[y_{i j}\right]\right)\right\|_{n} \leq \sum_{i, j=1}^{n} \varphi\left(x_{i j}, y_{i j}\right),
\end{aligned}
$$

for all $\left[x_{i j}\right],\left[y_{i j}\right] \in M_{n}(X)$. Then there exists a unique quartic mapping $Q: X \rightarrow Y$ satisfying

$$
\left\|f_{n}\left(\left[x_{i j}\right]\right)+f_{n}\left(-\left[x_{i j}\right]\right)-Q_{n}\left(\left[x_{i j}\right]\right)\right\|_{n} \leq \frac{L}{32-32 L} \sum_{i, j=1}^{n}\left(\varphi\left(x_{i j}, 0\right)+\varphi\left(-x_{i j}, 0\right)\right)
$$

for all $\left[x_{i j}\right] \in M_{n}(X)$.

Proof Setting $n=1$ in (2.2), we get

$$
\|D f(x, y)\| \leq \varphi(x, y) .
$$


Letting $y=0$ in (2.4), we get

$$
\|2 f(2 x)-24 f(x)-8 f(-x)\| \leq \varphi(x, 0)
$$

for all $x \in X$. Replacing $x$ by $-x$ in (2.5), we get

$$
\|2 f(-2 x)-24 f(-x)-8 f(x)\| \leq \varphi(x, 0)
$$

for all $x \in X$. Consider the set

$$
S:=\{g: X \rightarrow Y, g(0)=0\}
$$

and introduce the generalized metric on $S$ :

$$
d(g, h)=\inf \left\{K \in \mathbb{R}_{+}:\|g(x)-h(x)\| \leq K \varphi(x, 0)+K \varphi(-x, 0), \forall x \in X\right\} .
$$

It is easy to show that $(S, d)$ is complete (see [8, Theorem 2.5]).

Now we consider the linear mapping $J: S \rightarrow S$ such that

$$
J g(x):=16 g\left(\frac{x}{2}\right)
$$

for all $x \in X$. It follows from [7, Theorem 3.1] that

$$
d(J g, J h) \leq L d(g, h)
$$

for all $g, h \in S$.

Let $g(x):=f(x)+f(-x)$ for all $x \in X$. Then $g: X \rightarrow Y$ is an even mapping. It follows from (2.5) and (2.6) that

$$
\|2 g(2 x)-32 g(x)\| \leq \varphi(x, 0)+\varphi(-x, 0)
$$

for all $x \in X$. So

$$
\left\|g(x)-16 g\left(\frac{x}{2}\right)\right\| \leq \varphi\left(\frac{x}{2}, 0\right)+\varphi\left(\frac{-x}{2}, 0\right) \leq \frac{L}{32}(\varphi(x, 0)+\varphi(-x, 0))
$$

for all $x \in X$. Hence $d(g, J g) \leq \frac{L}{32}$.

By Theorem 1.2, there exists a mapping $Q: X \rightarrow Y$ satisfying the following:

(1) $Q$ is a fixed point of $J$, i.e.,

$$
Q\left(\frac{x}{2}\right)=\frac{1}{16} Q(x)
$$

for all $x \in X$. Then $Q: X \rightarrow Y$ is an even mapping. The mapping $Q$ is a unique fixed point of $J$ in the set

$$
M=\{g \in S: d(f, g)<\infty\}
$$


This implies that $Q$ is a unique mapping satisfying (2.7) such that there exists a $K \in(0, \infty)$ satisfying

$$
\|g(x)-Q(x)\| \leq K(\varphi(x, 0)+\varphi(-x, 0))
$$

for all $x \in X$.

(2) $d\left(J^{n} g, Q\right) \rightarrow 0$ as $m \rightarrow \infty$. This implies the equality

$$
\lim _{m \rightarrow \infty} 16^{m} g\left(\frac{x}{2}\right)=Q(x)
$$

for all $x \in X$.

(3) $d(g, Q) \leq \frac{1}{1-L} d(g, g g)$, which implies the inequality

$$
d(g, Q) \leq \frac{L}{32-32 L} .
$$

This implies that the inequality (2.3) holds.

It follows from (2.1), (2.2) and (2.8) that

$$
\begin{aligned}
\|D Q(x, y)\| & =\lim _{m \rightarrow \infty} 16^{m}\left\|D g\left(\frac{x}{2^{m}}, \frac{y}{2^{m}}\right)\right\| \\
& \leq \lim _{m \rightarrow \infty} 16^{m}\left(\varphi\left(\frac{x}{2^{m}}, \frac{y}{2^{m}}\right)+\varphi\left(-\frac{x}{2^{m}},-\frac{y}{2^{m}}\right)\right)=0
\end{aligned}
$$

for all $x, y \in X$. So $D Q(x, y)=0$ for all $x, y \in X$. Since $Q: X \rightarrow Y$ is even, the mapping $Q: X \rightarrow Y$ is a quartic mapping.

By Lemma 1.3, there exists a unique quartic mapping $Q: X \rightarrow Y$ satisfying (2.3), as desired.

Example 2.2 Let $\varphi: \mathbb{R}^{2} \rightarrow[0, \infty)$ be a function defined by

$$
\varphi(x)= \begin{cases}0, & \text { if } x=0, \\ \zeta x^{4}, & \text { if }|x|<1, \\ \zeta, & \text { otherwise }\end{cases}
$$

where $\zeta>0$ is a constant. Define a function $f_{q}: \mathbb{R} \rightarrow \mathbb{R}$ by

$$
f_{q}(x)=\sum_{n=0}^{\infty} \frac{\varphi\left(2^{n} x\right)}{16^{n}} .
$$

Then $f_{q}$ satisfies the functional inequality

$$
\left|D f_{q}(x, y)\right| \leq \frac{28 \times 16 \zeta}{15}\left(|x|^{4}+|y|^{4}\right)
$$


for all $x \in \mathbb{R}$. Let

$$
\begin{aligned}
& g_{q}(x)=f_{q}(x)+f_{q}(-x), \\
& g_{q}(x)=\zeta x^{4}, \\
& g_{q}(x / 2)=\frac{\zeta x^{4}}{2^{4}},
\end{aligned}
$$

for all $x \in \mathbb{R}$. We define the set $S=\left\{g_{q}: \mathbb{R} \rightarrow \mathbb{R}, g_{q}(0)=0\right\}$ and consider the generalized metric on $S$ as described in the proof of the above theorem. Also consider the mapping $J: S \rightarrow S$ such that

$$
J g_{q}(x)=16 g(x / 2)=g(x) .
$$

Now

$$
\begin{aligned}
\lim _{m \rightarrow \infty} 16^{m} g(x / 2) & =\lim _{m \rightarrow \infty} 16^{m} \frac{\zeta x^{4}}{2^{4}} \\
& =\lim _{m \rightarrow \infty} 16^{m} \frac{\zeta x^{4}}{2^{4(1-m)}} \\
& =Q(x) .
\end{aligned}
$$

It is clear that

$$
Q(x / 2)=\frac{1}{16} Q(x) .
$$

Moreover, we have

$$
\begin{aligned}
\|g(x)-16 g(x / 2)\| & =\left\|\zeta x^{4}-16 \frac{\zeta x^{4}}{24}\right\| \\
& \leq \varphi\left(\frac{x}{2}, 0\right)+\varphi\left(\frac{-x}{2}, 0\right) \\
& \leq \frac{L}{32} \varphi(x, 0)+\varphi(-x, 0) .
\end{aligned}
$$

Hence

$$
d(g, L g) \leq \frac{L}{32} .
$$

Also we can show that

$$
d(g, Q) \leq \frac{1}{1-L} d(g, J g) .
$$

The above result implies the following:

$$
d(g, Q) \leq \frac{32}{32-32 L} .
$$

Therefore all the conditions are fulfilled and by Lemma 1.3, $Q: \mathbb{R} \rightarrow \mathbb{R}$ satisfies (2.3). 
Corollary 2.3 Let $p>4$ and $\phi \geq 0$ be real numbers and $f: X \rightarrow Y$ be a mapping such that

$$
\left\|D f_{n}\left(\left[x_{i j}\right],\left[y_{i j}\right]\right)\right\|_{n} \leq \phi \sum_{i, j=1}^{n}\left(\left\|x_{i j}\right\|^{p}+\left\|y_{i j}\right\|^{p}+\left\|x_{i j}\right\|^{\frac{p}{2}} \cdot\left\|y_{i j}\right\|^{\frac{p}{2}}\right)
$$

for all $\left[x_{i j}\right],\left[y_{i j}\right] \in M_{n}(X)$. Then there exists a unique quartic mapping $Q: X \rightarrow Y$ satisfying

$$
\left\|f_{n}\left(\left[x_{i j}\right]\right)+f_{n}\left(-\left[x_{i j}\right]\right)-Q_{n}\left(\left[x_{i j}\right]\right)\right\|_{n} \leq \frac{\phi}{2^{p}-16} \sum_{i, j=1}^{n}\left\|x_{i j}\right\|^{p}
$$

for all $\left[x_{i j}\right] \in M_{n}(X)$.

Proof The proof follows from Theorem 2.1 by taking $L=2^{4-p}$ and

$$
\varphi(x, y)=\phi\left(\|x\|^{p}+\|y\|^{p}+\|x\|^{\frac{p}{2}} \cdot\|y\|^{\frac{p}{2}}\right)
$$

for all $x, y \in X$.

Remark 2.4 Let $f: X \rightarrow Y$ be a mapping with $f(0)=0$ for which there exists a function $\varphi: X^{2} \rightarrow[0, \infty)$ satisfying $(2.2)$ and

$$
\sum_{i, j=1}^{n} \varphi\left(x_{i j}, y_{i j}\right) \leq 16 L \sum_{i, j=1}^{n} \varphi\left(\frac{x_{i j}}{2}, \frac{y_{i j}}{2}\right)
$$

for all $\left[x_{i j}\right],\left[y_{i j}\right] \in M_{n}(X)$ and for some $L$ with $0<L<1$. By a similar method to the proof of Theorem 2.1, one can show that there exists a unique quartic mapping $Q: X \rightarrow Y$ satisfying

$$
\left\|f_{n}\left(\left[x_{i j}\right]\right)+f_{n}\left(-\left[x_{i j}\right]\right)-Q_{n}\left(\left[x_{i j}\right]\right)\right\|_{n} \leq \frac{L}{32-32 L} \sum_{i, j=1}^{n}\left(\varphi\left(x_{i j}, 0\right)+\varphi\left(-x_{i j}, 0\right)\right)
$$

for all $\left[x_{i j}\right] \in M_{n}(X)$.

Similarly, one can obtain a similar result to Corollary 2.3: Let $0<p<4$ and $\phi \geq 0$ be real numbers and $f: X \rightarrow Y$ be a mapping satisfying (2.10). Then there exists a unique quartic mapping $Q: X \rightarrow Y$

$$
\left\|f_{n}\left(\left[x_{i j}\right]\right)+f_{n}\left(-\left[x_{i j}\right]\right)-Q_{n}\left(\left[x_{i j}\right]\right)\right\|_{n} \leq \frac{\phi}{16-2^{p}} \sum_{i, j=1}^{n}\left\|x_{i j}\right\|^{p}
$$

for all $\left[x_{i j}\right] \in M_{n}(X)$.

\section{Fixed points and Hyers-Ulam stability of a cubic and quartic functional equation: an odd case}

Using the fixed point method, we prove the Hyers-Ulam stability of the functional equation $D f(x, y)=0$ in matrix Banach algebras: an odd case. 
Theorem 3.1 Let $f: X \rightarrow Y$ be a mapping with $f(0)=0$ for which there exists a function $\varphi: X^{2} \rightarrow[0, \infty)$ such that there exists an $L<1$ satisfying $(2.2)$ and

$$
\sum_{i, j=1}^{n} \varphi\left(x_{i j}, y_{i j}\right) \leq \sum_{i, j=1}^{n} \frac{1}{8} L \varphi\left(2 x_{i j}, 2 y_{i j}\right)
$$

for all $\left[x_{i j}\right],\left[y_{i j}\right] \in M_{n}(X)$. Then there exists a unique cubic mapping $C: X \rightarrow Y$ satisfying

$$
\left\|f_{n}\left(\left[x_{i j}\right]\right)-f_{n}\left(-\left[x_{i j}\right]\right)-C_{n}\left(\left[x_{i j}\right]\right)\right\|_{n} \leq \frac{L}{16-16 L} \sum_{i, j=1}^{n}\left(\varphi\left(x_{i j}, 0\right)+\varphi\left(-x_{i j}, 0\right)\right)
$$

for all $\left[x_{i j}\right] \in M_{n}(X)$.

Proof Setting $n=1$ in (2.2), we get

$$
\|D f(x, y)\| \leq \varphi(x, y) .
$$

Letting $y=0$ in (3.3), we get

$$
\|2 f(2 x)-24 f(x)-8 f(-x)\| \leq \varphi(x, 0)
$$

for all $x \in X$. Replacing $x$ by $-x$ in (3.4), we get

$$
\|2 f(-2 x)-24 f(-x)-8 f(x)\| \leq \varphi(x, 0)
$$

for all $x \in X$.

Consider the set

$$
S:=\{g: X \rightarrow Y, g(0)=0\}
$$

and introduce the generalized metric on S:

$$
d(g, h)=\inf \left\{K \in \mathbb{R}_{+}:\|g(x)-h(x)\| \leq K \varphi(x, 0)+K \varphi(-x, 0), \forall x \in X\right\} .
$$

It is easy to show that $(S, d)$ is complete (see [8, Theorem 2.5]).

Now we consider the linear mapping $J: S \rightarrow S$ such that

$$
J g(x):=16 g\left(\frac{x}{2}\right)
$$

for all $x \in X$. It follows from [7, Theorem 3.1] that

$$
d(J g, J h) \leq L d(g, h)
$$

for all $g, h \in S$. 
Let $g(x):=f(x)-f(-x)$ for all $x \in X$. Then $g: X \rightarrow Y$ is an odd mapping. It follows from (3.3) and (3.4) that

$$
\|2 g(2 x)-16 g(x)\| \leq \varphi(x, 0)+\varphi(-x, 0)
$$

for all $x \in X$. So

$$
\left\|g(x)-8 g\left(\frac{x}{2}\right)\right\| \leq \varphi\left(\frac{x}{2}, 0\right)+\varphi\left(-\frac{x}{2}, 0\right) \leq \frac{L}{16}(\varphi(x, 0)+\varphi(-x, 0))
$$

for all $x \in X$. Hence $d(g, J g) \leq \frac{L}{16}$.

By Theorem 1.2, there exists a mapping $C: X \rightarrow Y$ satisfying the following:

(1) $C$ is a fixed point of $J$, i.e.,

$$
C\left(\frac{x}{2}\right)=\frac{1}{8} C(x)
$$

for all $x \in X$. Then $C: X \rightarrow Y$ is an odd mapping. The mapping $C$ is a unique fixed point of $J$ in the set

$$
M=\{g \in S: d(f, g)<\infty\}
$$

This implies that $C$ is a unique mapping satisfying (3.6) such that there exists a $K \in(0, \infty)$ satisfying

$$
\|g(x)-C(x)\| \leq K(\varphi(x, 0)+\varphi(-x, 0))
$$

for all $x \in X$.

(2) $d\left(J^{n} g, C\right) \rightarrow 0$ as $m \rightarrow \infty$. This implies the equality

$$
\lim _{m \rightarrow \infty} 8^{m} g\left(\frac{x}{2^{m}}\right)=C(x)
$$

for all $x \in X$.

(3) $d(g, C) \leq \frac{L}{16-16 L}$, which implies the inequality (3.2) holds.

It follows from (3.1), (2.2) and (3.7) that

$$
\begin{aligned}
\|D C(x, y)\| & =\lim _{m \rightarrow \infty} 8^{m}\left\|D g\left(\frac{x}{2^{m}}, \frac{y}{2^{m}}\right)\right\| \\
& \leq \lim _{m \rightarrow \infty} 8^{m}\left(\varphi\left(\frac{x}{2^{m}}, \frac{y}{2^{m}}\right)+\varphi\left(-\frac{x}{2^{m}},-\frac{y}{2^{m}}\right)\right)=0,
\end{aligned}
$$

for all $x, y \in X$. So $D C(x, y)=0$ for all $x, y \in \mathrm{X}$. Since $C: X \rightarrow Y$ is odd, the mapping $C$ : $X \rightarrow Y$ is a cubic mapping. By Lemma 1.3, there exists a unique cubic mapping $C: X \rightarrow Y$ satisfying (3.2), as desired. 
Corollary 3.2 Let $p>3$ and $\phi \geq 0$ be real numbers and $f: X \rightarrow Y$ be a mapping satisfying (2.10). Then there exists a unique cubic mapping $C: X \rightarrow Y$ satisfying

$$
\left\|f_{n}\left(\left[x_{i j}\right]\right)-f_{n}\left(-\left[x_{i j}\right]\right)-C_{n}\left(\left[x_{i j}\right]\right)\right\|_{n} \leq \frac{\phi}{2^{p}-8} \sum_{i, j=1}^{n}\left\|x_{i j}\right\|^{p}
$$

for all $\left[x_{i j}\right] \in M_{n}(X)$.

Proof The proof follows from Theorem 3.1 by taking $L=2^{3-p}$ and

$$
\varphi(x, y)=\phi\left(\|x\|^{p}+\|y\|^{p}+\|x\|^{\frac{p}{2}} \cdot\|y\|^{\frac{p}{2}}\right)
$$

for all $x, y \in X$.

Combining Corollaries 2.3 and 3.2, we get the following.

Theorem 3.3 Let $p>4$ and $\phi \geq 0$ be real numbers and $f: X \rightarrow Y$ be a mapping satisfying (2.10). Then there exist a unique quartic mapping $Q: X \rightarrow Y$ and a unique cubic mapping $C: X \rightarrow Y$ satisfying

$$
\left\|2 f_{n}\left(\left[x_{i j}\right]\right)-Q_{n}\left(\left[x_{i j}\right]\right)-C_{n}\left(\left[x_{i j}\right]\right)\right\|_{n} \leq\left(\frac{1}{2^{p}-16}+\frac{1}{2^{p}-8}\right) \phi \sum_{i, j=1}^{n}\left\|x_{i j}\right\|^{p}
$$

for all $\left[x_{i j}\right] \in M_{n}(X)$.

Remark 3.4 Let $f: X \rightarrow Y$ be a mapping with $f(0)=0$ for which there exists a function $\varphi: X^{2} \rightarrow[0, \infty)$ satisfying $(2.2)$ and

$$
\sum_{i, j=1}^{n} \varphi\left(x_{i j}, y_{i j}\right) \leq 8 L \sum_{i, j=1}^{n} \varphi\left(\frac{x_{i j}}{2}, \frac{y_{i j}}{2}\right)
$$

for all $\left[x_{i j}\right],\left[y_{i j}\right] \in M_{n}(X)$ and for some $L$ with $0<L<1$. By a similar method to the proof of Theorem 3.1, one can show that there exists a unique cubic mapping $C: X \rightarrow Y$ satisfying

$$
\left\|f_{n}\left(\left[x_{i j}\right]\right)-f_{n}\left(-\left[x_{i j}\right]\right)-Q_{n}\left(\left[x_{i j}\right]\right)\right\|_{n} \leq \frac{L}{16-16 L} \sum_{i, j=1}^{n}\left(\varphi\left(x_{i j}, 0\right)+\varphi\left(-x_{i j}, 0\right)\right)
$$

for all $\left[x_{i j}\right] \in M_{n}(X)$.

Similarly, one can obtain a similar result to Corollary 3.2: Let $0<p<3$ and $\phi \geq 0$ be real numbers and $f: X \rightarrow Y$ be a mapping satisfying (2.10). Then there exists a unique cubic mapping $C: X \rightarrow Y$ satisfying

$$
\| f_{n}\left(\left[x_{i j}\right]\right)-f_{n}\left(-\left[x_{i j]}\right)-Q_{n}\left(\left[x_{i j}\right]\right)\left\|_{n} \leq \frac{\phi}{8-2^{p}} \sum_{i, j=1}^{n}\right\| x_{i j} \|^{p}\right.
$$

for all $\left[x_{i j}\right] \in M_{n}(X)$. 
Combining Remarks 2.4 and 3.4, we get the following.

Theorem 3.5 Let $0<p<3$ and $\phi \geq 0$ be real numbers and $f: X \rightarrow Y$ be a mapping satisfying (2.10). Then there exist a unique quartic mapping $Q: X \rightarrow Y$ and a unique cubic mapping $C: X \rightarrow Y$ satisfying

$$
\left\|2 f_{n}\left(\left[x_{i j}\right]\right)-Q_{n}\left(\left[x_{i j}\right]\right)-C_{n}\left(\left[x_{i j}\right]\right)\right\|_{n} \leq\left(\frac{1}{16-2^{p}}+\frac{1}{8-2^{p}}\right) \phi \sum_{i, j=1}^{n}\left\|x_{i j}\right\|^{p}
$$

for all $\left[x_{i j}\right] \in M_{n}(X)$.

\section{Fixed points and Hyers-Ulam stability of an additive and quartic functional equation: an even case}

One can easily show that an even mapping $f: X \rightarrow Y$ satisfies (1.4) if and only if the even mapping $f: X \rightarrow Y$ is a quartic mapping, i.e.,

$$
f(2 x+y)+f(2 x-y)=4 f(x+y)+4 f(x-y)+24 f(x)-6 f(y)
$$

and that an odd mapping $f: X \rightarrow Y$ satisfies (1.4) if and only if the odd mapping $f: X \rightarrow Y$ is an additive mapping, i.e.,

$$
f(x+y)=f(x)+f(y)
$$

It is easy to show that the function $f(x)=a x+b x^{4}$ satisfies the functional equation (1.4).

For a given mapping $f: X \rightarrow Y$, we define

$$
\begin{aligned}
C f(x, y):= & f(2 x+y)+f(2 x-y)-2 f(x+y)-2 f(-x-y)-2 f(x-y) \\
& -2 f(y-x)-14 f(x)-10 f(-x)+3 f(y)+3 f(-y)
\end{aligned}
$$

for all $x, y \in \mathrm{X}$.

Using the fixed point method, we prove the Hyers-Ulam stability of the functional equation $C f(x, y)=0$ in matrix Banach algebras: an even case.

Theorem 4.1 Let $f: X \rightarrow Y$ be a mapping with $f(0)=0$ for which there exists a function $\varphi: X^{2} \rightarrow[0, \infty)$ such that there exists an $L<1$ such that

$$
\begin{aligned}
& \sum_{i, j=1}^{n} \varphi\left(x_{i j}, y_{i j}\right) \leq \sum_{i, j=1}^{n} \frac{L}{16} \varphi\left(2 x_{i j}, 2 y_{i j}\right), \\
& \left\|C f_{n}\left(\left[x_{i j}\right],\left[y_{i j}\right]\right)\right\|_{n} \leq \sum_{i, j=1}^{n} \varphi\left(x_{i j}, y_{i j}\right),
\end{aligned}
$$

for all $\left[x_{i j}\right],\left[y_{i j}\right] \in M_{n}(X)$. Then there exists a unique quartic mapping $Q: X \rightarrow Y$ satisfying

$$
\left\|f_{n}\left(\left[x_{i j}\right]\right)+f_{n}\left(-\left[x_{i j}\right]\right)-Q_{n}\left(\left[x_{i j}\right]\right)\right\|_{n} \leq \frac{L}{32-32 L} \sum_{i, j=1}^{n}\left(\varphi\left(x_{i j}, 0\right)+\varphi\left(-x_{i j}, 0\right)\right)
$$

for all $\left[x_{i j}\right] \in M_{n}(X)$. 
Proof Setting $n=1$ in (4.2), we have

$$
\|D f(x, y)\| \leq \varphi(x, y)
$$

for all $x, y \in X$. Letting $y=0$ in (4.4), we obtain

$$
\|2 f(2 x)-18 f(x)-14 f(-x)\| \leq \varphi(x, 0)
$$

for all $x \in X$. Replacing $x$ by $-x$ in (4.5), we get

$$
\|2 f(-2 x)-18 f(-x)-14 f(x)\| \leq \varphi(-x, 0)
$$

for all $x \in X$.

Consider the set

$$
S:=\{g: X \rightarrow Y, g(0)=0\}
$$

and introduce the generalized metric on $S$ :

$$
d(g, h)=\inf \left\{K \in \mathbb{R}_{+}:\|g(x)-h(x)\| \leq K \varphi(x, 0)+K \varphi(-x, 0), \forall x \in X\right\} .
$$

It is easy to show that $(S, d)$ is complete (see [8, Theorem 2.5]).

Now we consider the linear mapping $J: S \rightarrow S$ such that

$$
J g(x):=16 g\left(\frac{x}{2}\right)
$$

for all $x \in X$. It follows from the proof of [7, Theorem 3.1] that

$$
d(J g, J h) \leq L d(g, h)
$$

for all $g, h \in S$.

Let $g(x):=f(x)+f(x)$ for all $x \in X$. Then $g: X \rightarrow Y$ is an even mapping. It follows from (4.5) and (4.6) that

$$
\|2 g(2 x)-32 g(x)\| \leq \varphi(x, 0)+\varphi(-x, 0)
$$

for all $x \in X$. So

$$
\| g(x)-16 g\left(\frac{x}{2}\right) \leq \varphi\left(\frac{x}{2}, 0\right)+\varphi\left(-\frac{x}{2}, 0\right) \leq \frac{L}{32}(\varphi(x, 0)+\varphi(-x, 0))
$$

for all $x \in X$. Hence $d(g, J g) \leq \frac{L}{32}$.

The rest of the proof is similar to the proof of Theorem 2.1.

Corollary 4.2 Let $p>4$ and $\phi \geq 0$ be real numbers and $f: X \rightarrow Y$ be a mapping such that

$$
\left\|C f_{n}\left(\left[x_{i j}\right],\left[y_{i j}\right]\right)\right\|_{n} \leq \phi \sum_{i, j=1}^{n}\left(\left\|x_{i j}\right\|^{p}+\left\|y_{i j}\right\|^{p}+\left\|x_{i j}\right\|^{\frac{p}{2}} \cdot\left\|y_{i j}\right\|^{\frac{p}{2}}\right)
$$


for all $\left[x_{i j}\right],\left[y_{i j}\right] \in M_{n}(X)$. Then there exists a unique quartic mapping $Q: X \rightarrow Y$ satisfying

$$
\left\|f_{n}\left(\left[x_{i j}\right]\right)+f_{n}\left(-\left[x_{i j}\right]\right)-Q_{n}\left(\left[x_{i j}\right]\right)\right\|_{n} \leq \frac{\phi}{2^{p}-16} \sum_{i, j=1}^{n}\left\|x_{i j}\right\|^{p}
$$

for all $\left[x_{i j}\right] \in M_{n}(X)$.

Proof The proof follows from Theorem 4.1 by taking $L=2^{4-p}$ and

$$
\varphi(x, y)=\phi\left(\|x\|^{p}+\|y\|^{p}+\|x\|^{\frac{p}{2}} \cdot\|y\|^{\frac{p}{2}}\right)
$$

for all $x, y \in X$.

Remark 4.3 Let $f: X \rightarrow Y$ be a mapping with $f(0)=0$ for which there exists a function $\varphi: X^{2} \rightarrow[0, \infty)$ satisfying (4.2) and

$$
\sum_{i, j=1}^{n} \varphi\left(x_{i j}, y_{i j}\right) \leq \sum_{i, j=1}^{n} 16 L \varphi\left(\frac{x_{i j}}{2}, \frac{y_{i j}}{2}\right)
$$

for all $\left[x_{i j}\right],\left[y_{i j}\right] \in M_{n}(X)$ and for some $L$ with $0<L<1$. By a similar method to the proof of Theorem 4.1, one can show that there exists a unique quartic mapping $Q: X \rightarrow Y$ satisfying

$$
\left\|f_{n}\left(\left[x_{i j}\right]\right)+f_{n}\left(-\left[x_{i j}\right]\right)-Q_{n}\left(\left[x_{i j}\right]\right)\right\|_{n} \leq \frac{1}{32-32 L} \sum_{i, j=1}^{n}\left(\varphi\left(x_{i j}, 0\right)+\varphi\left(-x_{i j}, 0\right)\right)
$$

for all $\left[x_{i j}\right] \in M_{n}(X)$. Similarly, one can obtain a similar result to Corollary 4.2: Let $0<p<4$ and $\phi \geq 0$ be real numbers and $f: X \rightarrow Y$ be a mapping satisfying (4.7). Then there exists a unique quartic mapping $Q: X \rightarrow Y$

$$
\left\|f_{n}\left(\left[x_{i j}\right]\right)+f_{n}\left(-\left[x_{i j}\right]\right)-Q_{n}\left(\left[x_{i j}\right]\right)\right\|_{n} \leq \frac{\phi}{16-2^{p}} \sum_{i, j=1}^{n}\left\|x_{i j}\right\|^{p}
$$

for all $\left[x_{i j}\right] \in M_{n}(X)$.

\section{Fixed points and Hyers-Ulam stability of an additive and quartic functional equation: an odd case}

Using the fixed point method, we prove the Hyers-Ulam stability of the functional equation $C f(x, y)=0$ in matrix Banach algebras: an odd case.

Theorem 5.1 Let $f: X \rightarrow Y$ be a mapping with $f(0)=0$ for which there exists a function $\varphi: X^{2} \rightarrow[0, \infty)$ satisfying (4.2) such that there exists an $L<1$ such that

$$
\sum_{i, j=1}^{n} \varphi\left(x_{i j}, y_{i j}\right) \leq \sum_{i, j=1}^{n} \frac{1}{2} L \varphi\left(2 x_{i j}, 2 y_{i j}\right)
$$


for all $\left[x_{i j}\right],\left[y_{i j}\right] \in M_{n}(X)$. Then there exists a unique additive mapping $A: X \rightarrow Y$ satisfying

$$
\left\|f_{n}\left(\left[x_{i j}\right]\right)-f_{n}\left(-\left[x_{i j}\right]\right)-A_{n}\left(\left[x_{i j}\right]\right)\right\|_{n} \leq \frac{L}{4-4 L} \sum_{i, j=1}^{n}\left(\varphi\left(x_{i j}, 0\right)+\varphi\left(-x_{i j}, 0\right)\right)
$$

$\left[x_{i j}\right] \in M_{n}(X)$.

Proof Setting $n=1$ in (4.2), we get

$$
\|C f(x, y)\| \leq \varphi(x, y)
$$

for all $x, y \in X$. Letting $y=0$ in (5.2), we have

$$
\|2 f(2 x)-18 f(x)-14 f(-x)\| \leq \varphi(x, 0)
$$

for all $x \in X$. Replacing $x$ by $-x$ in (5.3), we obtain

$$
\|2 f(-2 x)-18 f(-x)-14 f(x)\| \leq \varphi(x, 0)
$$

for all $x \in X$.

Consider the set

$$
S:=\{g: X \rightarrow Y, g(0)=0\}
$$

and introduce the generalized metric on $S$ :

$$
d(g, h)=\inf \left\{K \in \mathbb{R}_{+}:\|g(x)-h(x)\| \leq K \varphi(x, 0)+K \varphi(-x, 0), \forall x \in X\right\} .
$$

It is easy to show that $(S, d)$ is complete (see [8, Theorem 2.5]).

Now we consider the linear mapping $J: S \rightarrow S$ such that

$$
J g(x):=2 g\left(\frac{x}{2}\right)
$$

for all $x \in X$. It follows from the proof of [7, Theorem 3.1] that

$$
d(J g, J h) \leq L d(g, h)
$$

for all $g, h \in S$.

Let $g(x):=f(x)-f(-x)$ for all $x \in X$. Then $g: X \rightarrow Y$ is an odd mapping. It follows from (5.3) and (5.4) that

$$
\|2 g(2 x)-4 g(x)\| \leq \varphi(x, 0)+\varphi(-x, 0)
$$

for all $x \in X$. So

$$
\| g(x)-2 g\left(\frac{x}{2}\right) \leq \varphi\left(\frac{x}{2}, 0\right)+\varphi\left(-\frac{x}{2}, 0\right) \leq \frac{L}{4}(\varphi(x, 0)+\varphi(-x, 0))
$$

for all $x \in X$. Hence $d(g, g g) \leq \frac{L}{4}$. 
The rest of the proof is similar to the proofs of Theorems 2.1 and 3.1.

Example 5.2 Let $\varphi: \mathbb{R}^{2} \rightarrow[0, \infty)$ be a function defined by

$$
\varphi(x)= \begin{cases}0, & \text { if } x=0 \\ \zeta x, & \text { if }|x|<1 \\ \zeta, & \text { otherwise }\end{cases}
$$

where $\zeta>0$ is a constant. Define a function $f_{q}: \mathbb{R} \rightarrow \mathbb{R}$ by

$$
f_{q}(x)=\sum_{n=0}^{\infty} \frac{\varphi\left(2^{n} x\right)}{2^{n}}
$$

Then $f_{q}$ satisfies the functional inequality

$$
\left|D f_{q}(x, y)\right| \leq 56 \zeta(|x|+|y|)
$$

for all $x \in \mathbb{R}$. By the same procedure as in Example 2.2, we can find a mapping $A_{q}$ satisfying the inequality (5.1).

Corollary 5.3 Let $p>3$ and $\phi \geq 0$ be real numbers and $f: X \rightarrow Y$ be a mapping satisfying (4.7). Then there exists a unique cubic mapping $A: X \rightarrow Y$ satisfying

$$
\left\|f_{n}\left(\left[x_{i j}\right]\right)-f_{n}\left(-\left[x_{i j}\right]\right)-A_{n}\left(\left[x_{i j}\right]\right)\right\|_{n} \leq \frac{\phi}{2^{p}-2} \sum_{i, j=1}^{n}\left\|x_{i j}\right\|^{p}
$$

for all $\left[x_{i j}\right] \in M_{n}(X)$.

Proof The proof follows from Theorem 5.1 by taking $L=2^{1-p}$ and

$$
\varphi(x, y)=\phi\left(\|x\|^{p}+\|y\|^{p}+\|x\|^{\frac{p}{2}} \cdot\|y\|^{\frac{p}{2}}\right)
$$

for all $x, y \in X$.

Combining Corollaries 4.2 and 5.3, we get the following.

Theorem 5.4 Let $p>4$ and $\phi \geq 0$ be real numbers and $f: X \rightarrow Y$ be a mapping satisfying (4.7). Then there exist a unique quartic mapping $Q: X \rightarrow Y$ and a unique additive mapping $A: X \rightarrow Y$ satisfying

$$
\left\|2 f_{n}\left(\left[x_{i j}\right]\right)-Q_{n}\left(\left[x_{i j}\right]\right)-C_{n}\left(\left[x_{i j}\right]\right)\right\|_{n} \leq\left(\frac{1}{2^{p}-16}+\frac{1}{2^{p}-2}\right) \phi \sum_{i, j=1}^{n}\left\|x_{i j}\right\|^{p}
$$

for all $\left[x_{i j}\right] \in M_{n}(X)$. 
Remark 5.5 Let $f: X \rightarrow Y$ be a mapping with $f(0)=0$ for which there exists a function $\varphi: X^{2} \rightarrow[0, \infty)$ satisfying $(4.2)$ and

$$
\sum_{i, j=1}^{n} \varphi\left(x_{i j}, y_{i j}\right) \leq \sum_{i, j=1}^{n} \varphi\left(\frac{x_{i j}}{2}, \frac{y_{i j}}{2}\right)
$$

for all $\left[x_{i j}\right],\left[y_{i j}\right] \in M_{n}(X)$ and for some $L$ with $0<L<1$. By a similar method to the proof of Theorem 5.1, one can show that there exists a unique additive mapping $A: X \rightarrow Y$ satisfying

$$
\left\|f_{n}\left(\left[x_{i j}\right]\right)-f_{n}\left(-\left[x_{i j}\right]\right)-A_{n}\left(\left[x_{i j}\right]\right)\right\|_{n} \leq \frac{1}{4-4 L} \sum_{i, j=1}^{n}\left(\varphi\left(x_{i j}, 0\right)+\varphi\left(-x_{i j}, 0\right)\right)
$$

for all $\left[x_{i j}\right] \in M_{n}(X)$.

Similarly, one can obtain a similar result to Corollary 5.3: Let $0<p<3$ and $\phi \geq 0$ be real numbers and $f: X \rightarrow Y$ be a mapping satisfying (4.7). Then there exists a unique additive mapping $A: X \rightarrow Y$ satisfying

$$
\left\|f_{n}\left(\left[x_{i j}\right]\right)+f_{n}\left(-\left[x_{i j}\right]\right)-A_{n}\left(\left[x_{i j}\right]\right)\right\|_{n} \leq \frac{\phi}{2-2^{p}} \sum_{i, j=1}^{n}\left\|x_{i j}\right\|^{p}
$$

for all $\left[x_{i j}\right] \in M_{n}(X)$.

Combining Remarks 4.3 and 5.5, we get the following.

Theorem 5.6 Let $0<p<1$ and $\phi \geq 0$ be real numbers and $f: X \rightarrow Y$ be a mapping satisfying (4.7). Then there exist a unique quartic mapping $Q: X \rightarrow Y$ and a unique additive mapping $A: X \rightarrow Y$ satisfying

$$
\left\|2 f_{n}\left(\left[x_{i j}\right]\right)-Q_{n}\left(\left[x_{i j}\right]\right)-A_{n}\left(\left[x_{i j}\right]\right)\right\|_{n} \leq\left(\frac{1}{16-2^{p}}+\frac{1}{2-2^{p}}\right) \phi \sum_{i, j=1}^{n}\left\|x_{i j}\right\|^{p}
$$

for all $\left[x_{i j}\right] \in M_{n}(X)$.

\section{Acknowledgements}

The authors thank the Basque Government for its support of this work through Grant IT1207-19. We would like to express our gratitude to the anonymous referees for their helpful suggestions and corrections.

\section{Funding}

This study is supported financially by the Basque Government through Grant IT1207-19.

Availability of data and materials

Not applicable.

Competing interests

The authors declare that they have no competing interests.

\section{Authors' contributions}

The authors equally conceived of the study, participated in its design and coordination, drafted the manuscript, participated in the sequence alignment, and read and approved the final manuscript. 


\section{Author details}

1 Department of Mathematical Sciences, Fatima Jinnah Women University, Rawalpindi, Islamic Republic of Pakistan.

${ }^{2}$ Department of Mathematics, International Islamic University, Islamabad, Islamic Republic of Pakistan. ${ }^{3}$ Department of Mathematics, Faculty of Science, Ege University, Bornova, 35100, Izmir, Turkey. ${ }^{4}$ Institute of Research and Development of Processes, University of the Basque Country, 48940 Leioa, Spain.

\section{Publisher's Note}

Springer Nature remains neutral with regard to jurisdictional claims in published maps and institutional affiliations.

Received: 6 August 2020 Accepted: 18 November 2020 Published online: 25 November 2020

\section{References}

1. Aoki, T.: On the stability of the linear transformation in Banach spaces. J. Math. Soc. Jpn. 2, 64-66 (1950)

2. Ayoubi, M., Zeglami, D.: D'Alembert's functional equations on monoids with an anti-endomorphism. Results Math 75(2), Article ID 74 (2020)

3. Badora, R:: The Ulam stability problem for the functional equation $f(x \star g(y))=f(x) f(y)$. Results Math. 75(2), Article ID $62(2020)$

4. Baradol, P., Gopal, D., Radenovic, S.: Computational fixed points in graphical rectangular metric spaces with application. J. Comput. Appl. Math. 375, 112805 (2020)

5. Bourgin, D.G.: Classes of transformations and bordering transformations. Bull. Am. Math. Soc. 57, 223-237 (1951)

6. Budhia, L., Aydi, H., Ansari, A.H., Gopal, D.: Some new fixed point results in rectangular metric spaces with an application to fractional-order functional differential equations. Nonlinear Anal., Model. Control 25(4), 580-597 (2020)

7. Cadariu, L., Radu, V.: Fixed points and the stability of Jensen's functional equation. J. Inequal. Pure Appl. Math. 4(1), Article ID 4 (2003)

8. Cadariu, L., Radu, V.: On the stability of the Cauchy functional equation: a fixed point approach. Grazer Math. Ber. 346 43-52 (2004)

9. Cadariu, L., Radu, V.: Fixed point methods for the generalized stability of functional equations in a single variable. Fixed Point Theory Appl. 2008, Article ID 749392 (2008)

10. Cholewa, P.W.: Remarks on the stability of functional equations. Aequ. Math. 27, 76-86 (1984)

11. Czerwik, S.: On the stability of the quadratic mapping in normed spaces. Abh. Math. Semin. Univ. Hamb. 62, 59-64 (1992)

12. Diaz, J., Margolis, B.: A fixed point theorem of the alternative for contractions on a generalized complete metric space. Bull. Am. Math. Soc. 74, 305-309 (1968)

13. Găvruta, P.: A generalization of the Hyers-Ulam-Rassias stability of approximately additive mappings. J. Math. Anal. Appl. 184, 431-436 (1994)

14. Gopal, D., Abbas, M., Patel, D.K., Vetro, C.: Fixed points of $\alpha$-type F-contractive mappings with an application to nonlinear fractional differential equation. Acta Math. Sci. 36(3), 957-970 (2016)

15. Hyers, D.H.: On the stability of the linear functional equation. Proc. Natl. Acad. Sci. USA 27, 222-224 (1941)

16. Hyers, D.H., Isac, G., Rassias, Th.M.: Stability of Functional Equations in Several Variables. Birkhäuser, Basel (1998)

17. Isac, G., Rassias, Th.M.: Stability of $\psi$-additive mappings: applications to nonlinear analysis. Int. J. Math. Math. Sci. 19, 219-228 (1996)

18. Jun, K., Kim, H.: The generalized Hyers-Ulam-Rassias stability of a cubic functional equation. J. Math. Anal. Appl. 274, 867-878 (2002)

19. Lakzian, H., Gopal, D., Sintunavarat, W.: New fixed point results for mappings of contractive type with an application to nonlinear fractional differential equations. J. Fixed Point Theory Appl. 18, 251-266 (2016)

20. Lee, S., Im, S., Hwang, I.: Quartic functional equations. J. Math. Anal. Appl. 307, 387-394 (2005)

21. Park, C., Cho, Y., Han, M.: Functional inequalities associated with Jordan-von Neumann type additive functional equations. J. Inequal. Appl. 2007, Article ID 41820 (2007)

22. Park, C., Najati, A.: Homomorphisms and derivations in $C^{*}$-algebras. Abstr. Appl. Anal. 2007, Article ID 80630 (2007)

23. Radu, V.: The fixed point alternative and the stability of functional equations. Fixed Point Theory 4, $91-96$ (2003)

24. Rassias, J.M.: On approximation of approximately linear mappings by linear mappings. J. Funct. Anal. 46, 126-130 (1982)

25. Rassias, J.M.: Solution of the Ulam stability problem for quartic mappings. J. Indian Math. Soc. 67, 169-178 (2000)

26. Rassias, J.M.: Solution of the Ulam problem for cubic mappings. An. Univ. Vest. Timiš., Ser. Mat.-Inform. 38, 121-132 (2000)

27. Rassias, J.M.: Solution of the Ulam stability problem for cubic mappings. Glas. Mat. Ser. III 36(56), 63-72 (2001)

28. Rassias, Th.M.: On the stability of the linear mapping in Banach spaces. Proc. Am. Math. Soc. 72, 297-300 (1978)

29. Skof, F.: Propriet'a locali e approssimazione di operatori. Rend. Semin. Mat. Fis. Milano 53, 113-129 (1983)

30. Ulam, S.M.: Problems in Modern Mathematics. Wiley, New York (1960) 